Type of the Paper (Article)

\title{
Heat Shock Factor 1 directly regulates postsynaptic scaffolding PSD-95 and controls striatal synaptic density in aging and Huntington's disease
}

\author{
Nicole Zarate ${ }^{1}$, Taylor A. Intihar ${ }^{1}$, Dahyun Yu ${ }^{1}$, Jacob Sawyer ${ }^{1}$, Wei Tsai ${ }^{1}$, Maha Syed ${ }^{1}$, Luke Carlson ${ }^{1}$ and Rocio \\ Gomez-Pastor ${ }^{1, *}$
}
1 Department of Neuroscience, University of Minnesota, School of Medicine, Minneapolis, MN, USA. zarat013@umn.edu (N.Z.), intih006@umn.edu (T.A.I.), Yu.Dahyun@mayo.edu (D.Y.), sawye271@umn.edu (J.S.), tsaix246@umn.edu (W.T.), syed0022@umn.edu (M.S.), carl5146@umn.edu (L.C.), rgomezpa@umn.edu (R.G-P)
* Correspondence: rgomezpa@umn.edu, Tel.: +1-612-626-6824

\begin{abstract}
PSD-95 (Dlg4) is an ionotropic glutamate receptor scaffolding protein essential in synapse stability and neurotransmission. PSD-95 levels are reduced during aging and in neurodegenerative diseases like Huntington's disease (HD), and it is believed to contribute to synaptic dysfunction and behavioral deficits. However, the mechanism responsible for PSD-95 dysregulation under these conditions is unknown. The Heat Shock transcription Factor 1 (HSF1), canonically known for its role in protein homeostasis, is also depleted in both aging and HD. Synaptic protein levels, including PSD-95, are influenced by alterations in HSF1 levels and activity, but the direct regulatory relationship between PSD-95 and HSF1 has yet to be determined. Here, we showed that HSF1 chronic or acute depletion in cell lines and mice decreased PSD-95 expression. Furthermore, $\mathrm{HSF}^{(+-)}$mice had reduced PSD-95 synaptic puncta that paralleled a loss in thalamo-striatal excitatory synapses, an important circuit disrupted early in HD. We demonstrated that HSF1 binds to regulatory elements present in the PSD-95 gene and directly regulates PSD-95 expression. HSF1 DNA-binding on the PSD-95 gene was disrupted in an age-dependent manner in WT mice and worsened in HD cells and mice, leading to reduced PSD-95 levels. These results demonstrate a direct role of HSF1 in synaptic gene regulation that has important implications in synapse maintenance in basal and pathological conditions.
\end{abstract}

Keywords: HSF1, PSD-95, aging, Huntington's disease

\section{Introduction}

The postsynaptic scaffolding protein PSD-95 $(D \lg 4)$ is a member of the membraneassociated guanylate kinase (MAGUK) family of proteins known for anchoring ionotropic glutamate receptors (AMPAR and NMDARs) to the membrane. Changes in the levels of PSD-95 alter clustering and maintenance of glutamate receptors, thus playing an essential role in regulating synaptic transmission and plasticity [1,2]. Glutamatergic synaptic transmission and plasticity are fundamental mechanisms contributing to memory and cognition. Transcriptional dysregulation of PSD-95 has been reported during aging and in several neurodegenerative diseases (NDs) including Alzheimer's (AD) and Huntington's disease (HD), which it is believed to contribute to alterations in synaptic function and behavioral deficits [3-6]. However, the mechanisms involved in the dysregulation of PSD-95 under these conditions are not fully understood. Previous studies have identified several transcriptional regulators of Dlg4 including the Ikaros 
family zinc finger transcription factor Eos and Early Growth Response 1 (Egr-1) [7-9]. Our understanding of the transcriptional regulation of PSD-95 primarily relies on neurodevelopmental studies where these regulators are expressed in abundance. However, the expression of some of these regulators decline in the adult brain $[7,8,10]$. Therefore, alternative unknown mechanisms for the regulation of PSD-95 might exist in the fully developed brain.

The Heat Shock Transcription Factor 1 (HSF1), traditionally known for its role in regulating stress response and protein homeostasis [11] has been recently proposed as a regulator of synapse stability and memory consolidation [5,11-14]. This is supported by studies showing basal as well as stress-dependent accumulation of various chaperones within synaptosomes (isolated synaptic terminals) where they participate in modulating synaptic protein homeostasis $[15,16]$. However, recent studies have suggested a more direct role of HSF1 in the regulation of various synaptic components. In line with these studies, $H_{s f} 1^{\mathrm{KO}}$ mice showed aberrant synapse formation in the hippocampus, altered expression of synaptic proteins like the polysialylated-neural cell adhesion molecule (PSA-NCAM) and PSD-95, and working memory deficits $[14,17,18]$. Additional genetic and pharmacological manipulations aimed at activating HSF1 under both basal and pathological conditions resulted in increased levels of select synaptic proteins including PSD-95, Synapsin I and Synaptophysin [5,17]. Therefore, it is reasonable to hypothesize that HSF1 plays a direct role in the regulation of synaptic function by directly controlling the expression of specific synaptic genes.

HSF1 protein levels decline during aging, a phenomenon that is exacerbated in different NDs like HD where HSF1 was reported to be abnormally degraded [19-23]. HD is a fatal neurodegenerative disorder manifested by motor and cognitive decline. HD is caused by a polyglutamine repeat expansion in the $\mathrm{Htt}$ gene, resulting in a mutant form of the HTT protein (mtHTT) prone to misfolding and aggregation [24] that preferentially affects neurons of the striatum. Interestingly, down-regulation of PSD-95 within the striatum is considered a pathological marker in HD and reflects synaptic dysfunction $[25,26]$. However, whether depletion of HSF1 in aging or HD directly contributes to PSD95 dysregulation and synaptic dysfunction has yet to be determined.

In this study, we sought to determine whether HSF1 is a direct regulator of PSD-95 expression. We focused our analyses in both immortalized striatal cells and striatum tissues given the role of this brain region in the regulation of motor control and working memory in both aging and HD, and in which changes of PSD-95 and HSF1 were previously reported [27-32]. We showed that PSD-95 and HSF1 protein levels decrease in parallel in the striatum of WT mice in an age-dependent manner, a phenomenon that is exacerbated in the zQ175 HD mouse model. We established proof of concept for the role of HSF1 in regulating PSD-95 expression and synapse stability by conducting chronic and acute depletion of HSF1 in striatal cells and mice which resulted in the downregulation of PSD-95 and loss of striatal excitatory synapse density. Finally, we demonstrated that HSF1 binds to regulatory elements (Heat Shock Elements, HSEs) present in both murine and human Dlg4 genes and that such binding decreased over time and in the presence of 
mtHTT, coinciding with transcriptional depletion of PSD-95. Overall, our data provides strong evidence for the regulatory role of HSF1 on PSD-95 expression and highlights the importance of this regulatory interaction in the maintenance of striatal glutamatergic synapses in both physiology and disease.

\section{Results}

\section{1. mHTT exacerbates an age-dependent depletion of PSD-95 and HSF1}

Previous studies showed that HSF1 and PSD-95 decreased in an age-dependent manner in mouse models as well as humans [4,11,33-35] although the relationship between the alterations in those proteins has not yet been established. We therefore investigated whether age-dependent dysregulation of HSF1 relates to PSD-95 depletion. We performed immunoblot analyses of HSF1 and PSD-95 in striatal tissue from WT mice at 3, 6, 12 and 22 months old (Fig. 1A-B). HSF1 protein levels significantly decreased between 3 and 6 months $(\sim 26 \%$ reduction, $\mathrm{p}=0.0011)$ and reached its lowest levels at 22 months $(\sim 46$

$\%$ reduction from 3 months, $\mathrm{p}=0.0008$ ). Similarly, PSD-95 levels decreased over time, with the lowest concentration at 22 months ( $\sim 54 \%$ reduction from 3 months, $\mathrm{p}=0.0008)$. These data recapitulate previous reports and indicate a parallel time-dependent depletion of these two proteins in the striatum of WT mice.

In HD, depletion of PSD-95 is particularly relevant for its role in dysregulation of striatal glutamatergic synapses. HD mouse models and postmortem tissue from patients with HD have shown reduced levels of PSD-95 in the striatum compared to control individuals [25,36-38]. We previously showed HSF1 is abnormally degraded in HD [19]. Given our observation that HSF1 reduction parallels PSD-95 depletion in WT animals over time, we explored whether HSF1 and PSD-95 levels were concomitantly reduced in HD. We used the heterozygous zQ175 HD mouse model [30] and performed immunohistochemical and immunoblotting analyses for PSD-95 (Fig. 1C-D). We examined the dorsolateral striatum of mice harvested at 5 weeks, a time point at which excitatory synapse deficits are already shown $[19,26]$, as well as 6 months, a time point characterized by significant mtHTT aggregation, the onset of motor symptoms, and global transcriptional deficits [30,39] (Fig. 1C). We observed that zQ175 PSD-95 intensity was significantly lower than in WT mice at 5 weeks $(1.6 \mathrm{E}+06$ vs $1.2 \mathrm{E}+06, \mathrm{p}=0.043)$ and further decreased at 6 months (1.1E+06 vs 7.4E+05, p=0.0028) (Fig 1D). Comparisons within genotypes also showed a significant depletion of PSD-95 over time that was exacerbated in zQ175 mice (1.2E+06 vs 7.4.0E+05, $\mathrm{p}=0.0051)$.

Immunoblot analyses of striatal tissue from 6 month WT and zQ175 mice confirmed a reduction in both HSF1 and PSD-95 in HD mice (Fig. 1E). Depletion of HSF1 in HD has been previously connected to post-translational events [19] but downregulation of PSD95 seems to be caused by transcriptional dysregulation. We confirmed that the depletion of PSD-95 protein levels are consistent at the transcript level in $\mathrm{zQ175}$ mice compared to WT based on RT-qPCR analyses of Dlg4 mRNA levels (Fig. 1F). Transcriptional dysregulation of Dlg4 was also seen in an immortalized striatal cell model of HD 


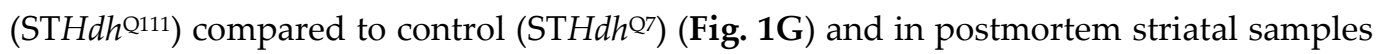
from patients with HD (Fig. 1H). These data demonstrated that PSD-95 depletion is sustained in an age-dependent manner alongside HSF1, a phenomenon that is exacerbated in HD.

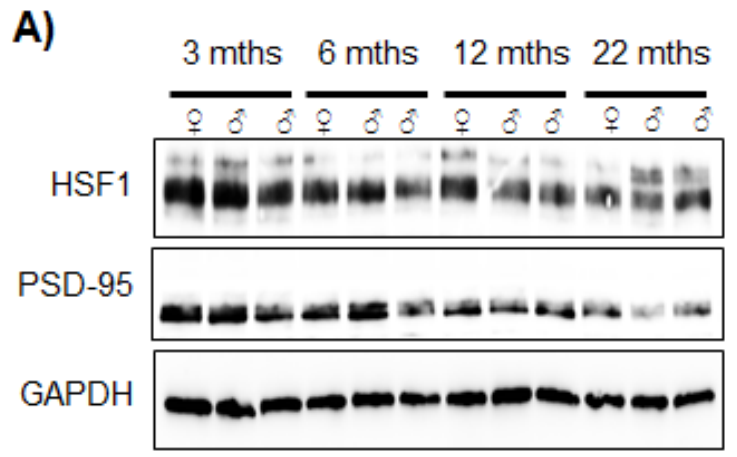

C)

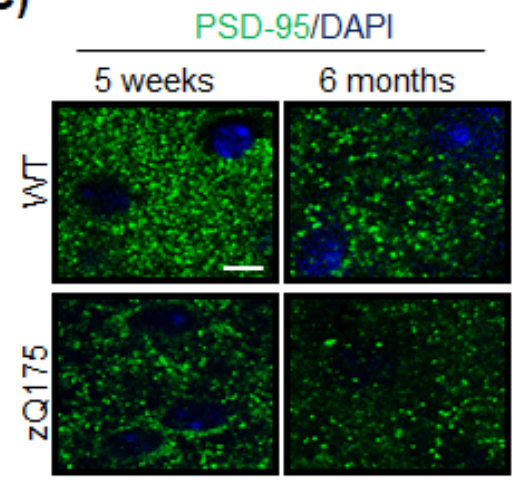

F)

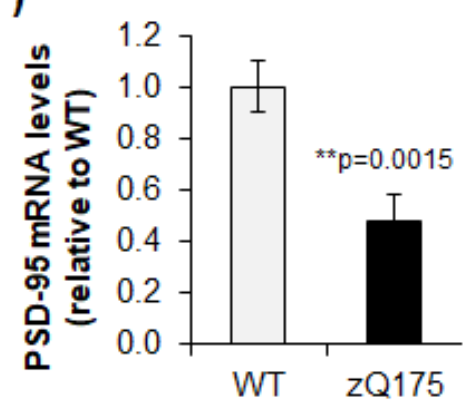

B)

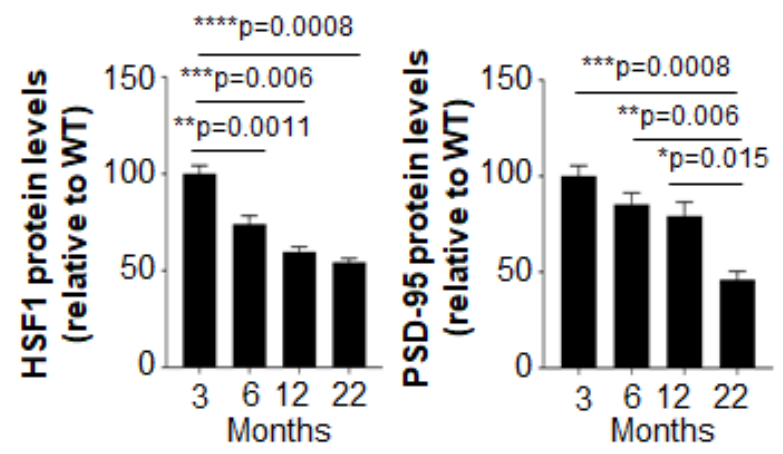

E)

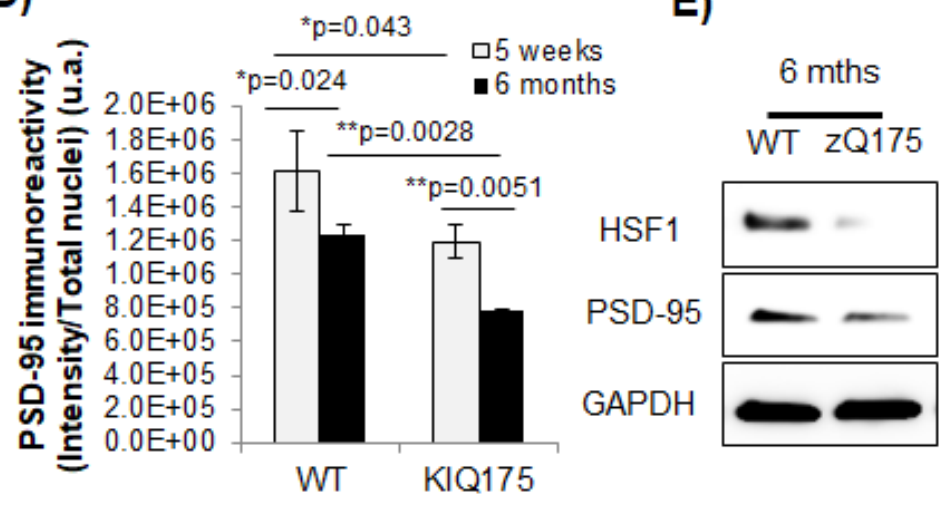

G)

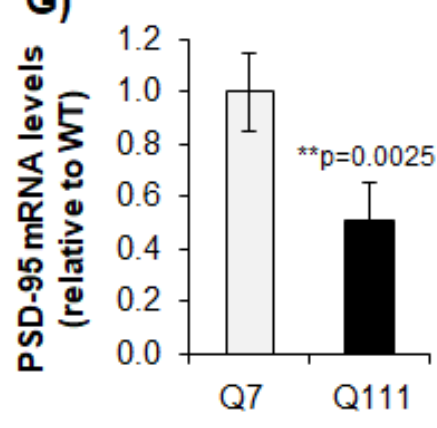

H)

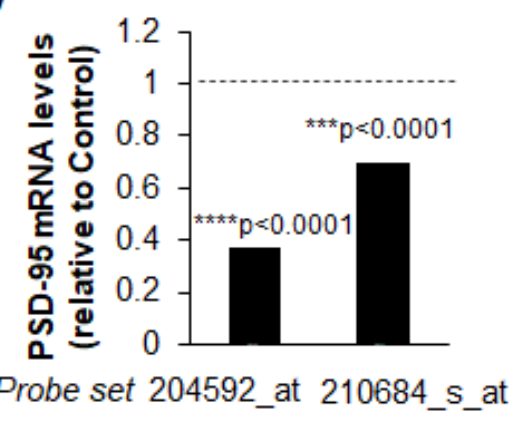

Figure 1. mtHTT exacerbates age-dependent down-regulation of HSF1 and PSD-95. A) Immunoblot for HSF1 and PSD95 from striatum samples from WT mice at different ages. GAPDH is used as a loading control. B) HSF1 and PSD-95 protein levels quantified from A using ImageJ analyses. Data was normalized to GAPDH levels and relativized to WT ( $n=3-5$ mice genotype). C) PSD-95 immunofluorescence in the striatum of WT and zQ175 mice. Scale bar 5 $\mu$ m. D) PSD-95 intensity quantified by ImageJ from images in C. ( $n=4$ mice/genotype). E) Representative immunoblotting for WT and zQ175 at 6 months ( $n=3$ mice/genotype). GAPDH was used as a loading control. F) PSD-95 mRNA levels in the striatum of WT $(n=5)$ and zQ175 $(n=6)$ mice at 6 months. G) PSD-95 mRNA levels in the caudate of patients with HD (n=44) relative to unaffected individuals $(n=36)$ obtained from Hodges et al., [6]. Data is shown as Log2 FC from two different probe sets. Error bars denote mean \pm SEM. One-way ANOVA with Tukey's post-hoc correction in B and unpaired Student's t-test in D, F, G. ${ }^{*} p<0.05,{ }^{* *} p<0.01,{ }^{* * *} \mathrm{p}<0.001,{ }^{* * * *} \mathrm{p}<0.0001$. Uncropped blots can be found in Supplementary Figure 1. 
We hypothesized that the depletion of HSF1 is responsible for the transcriptional dysregulation of Dlg4 in both aging and HD and therefore sought to characterize how direct modification of HSF1 levels influences Dlg4 expression. As proof of concept, STHdh ${ }^{\mathrm{Q} 7}$ cells were transfected with an siRNA targeting Hsf1 (siHsf1) followed by immunoblotting (Fig. 2A). Cells transfected with non-targeting siRNA (scr) were used as a negative control. HSF1 knockdown resulted in a $\sim 60 \%$ reduction in HSF1 protein levels $(\mathrm{p}=0.008)$ and was accompanied by a $\sim 23 \%$ reduction in PSD-95 protein levels $(\mathrm{p}=0.017$ )

(Fig. 2A-B).

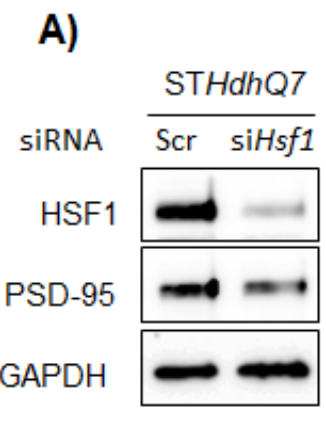

D)

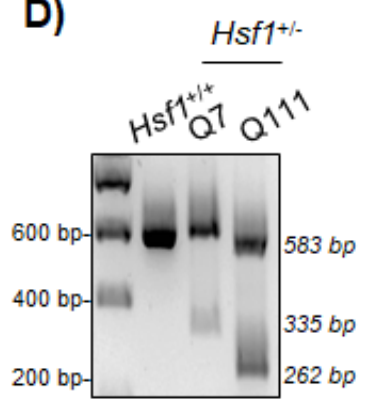

B)

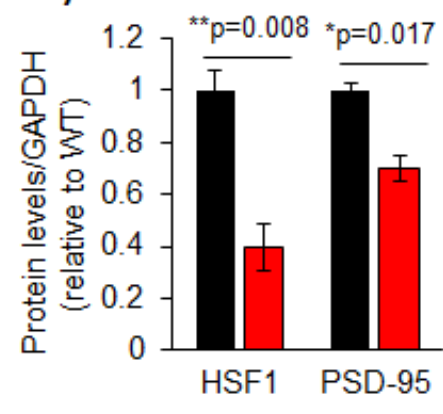

C)

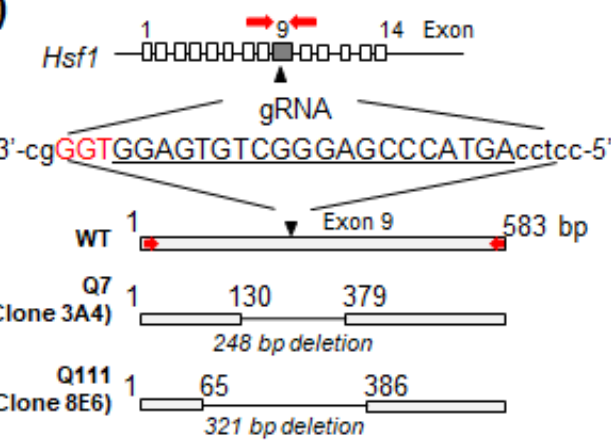

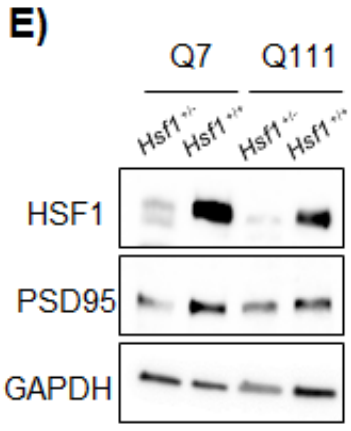

F)

HSF1

PSD-95
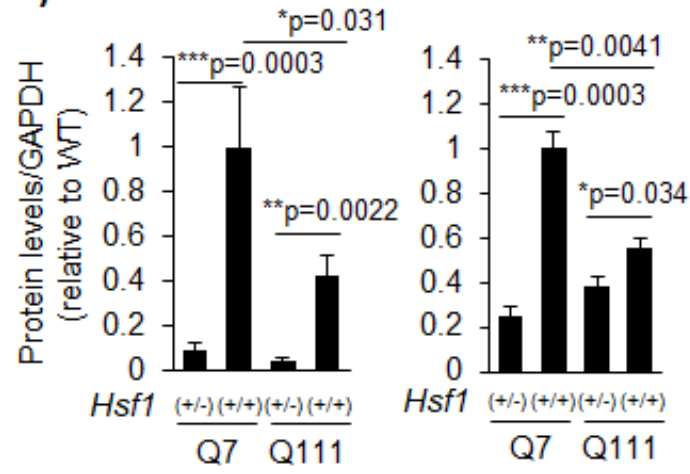

Figure 2. Acute or chronic depletion of HSF1 levels decreased PSD-95 levels in immortalized striatal cells. A) Immunoblot of STHdh ${ }^{\mathrm{Q} 7}$ cells transfected with non-targeting siRNA (scr.) or siHSF1. GAPDH is used as a loading control. B) HSF1 and PSD-95 protein levels quantified from A using ImageJ analyses. Data was normalized to GAPDH levels and relativized to WT (n=3). C) Genetic diagram representing the generation of HSF1 heterozygous (+/-) STHdh cell lines using CRISPR/Cas9. Guide RNA (gRNA) targets position +174 within exon 9 of HSF1. Cas 9 cleavage generated a $248 \mathrm{bp}$ deletion in STHdh ${ }^{\mathrm{Q7}}$ (clone 3A4) and a 321bp deletion in STHdh ${ }^{\mathrm{Q} 111}$ (clone 8E6). D) PCR analyses from genomic DNA obtained from WT, clone 3A4 and clone 8E6 for HSF1 exon 9 DNA region. E) Immunoblotting for WT and STHdh cell lines lacking one allele of HSF1. GAPDH was used as a loading control. F) HSF1 and PSD-95 protein levels quantified from A using ImageJ analyses. Data was normalized to GAPDH levels and relativized to WT $(n=3)$. Error bars denote mean \pm SEM. Unpaired Student's t-test. ${ }^{*} \mathrm{p}<0.05,{ }^{* *} \mathrm{p}<0.01,{ }^{* * *} \mathrm{p}<0.001$. Uncropped blots can be found in Supplementary material. Uncropped blots can be found in Supplementary Figure 2.

The use of siRNA showed that transient and acute depletion of HSF1 significantly impacted the levels of PSD-95. However in both aging and neurodegeneration, depletion of HSF1 occurs in a sustained manner. To better mimic this chronic depletion of HSF1, we generated Hsf1 heterozygous (+/-) cells for both STHdhQ7 and STHdhQ111 using the CRISPR/Cas9 system. The guide RNA (gRNA) was targeted to a region within exon 9 of the Hsf1 gene and single cell clones were isolated and genotyped. We selected clone 3A4 
for $\mathrm{STH} d h^{\mathrm{Q} 7}$ and clone $8 \mathrm{E} 6$ for STHdh ${ }^{\mathrm{Q} 111}$ cells containing a 248 bp and 321 bp deletion respectively in one allele of Hsf1 (Fig. 2C-D, Supplementary Fig. 1). Immunoblotting confirmed a significant reduction in the levels of HSF1 in both STHdh $h^{\mathrm{Q} 7}: H s f 1^{(+-)}(\mathrm{p}=0.0003)$ and STHdhQ111:Hsf1 ${ }^{(+)}(\mathrm{p}=0.0022)$ cells (Fig 2E-F). Chronic depletion of HSF1 in both STHdh cell lines showed a significant reduction in PSD-95 protein levels (Fig. 2G). Taken together, these data show that altering HSF1 levels by either acute and transient silencing with siRNA or by knocking-out one allele of HSF1 decrease of PSD-95 protein levels.

Due to the significant changes in PSD-95 levels when directly modulating HSF1 levels in vitro, we explored whether disruption of HSF1 in vivo would affect PSD-95 expression in a similar manner by using heterozygous $H s f 1^{(+-)}$mice. Immunoblot analyses of HSF1 and Hsp70, a well-known canonical target of HSF1 [11], showed a reduction in these two proteins in $\mathrm{Hsf1}^{(+-)}$mice compared to WT (HSF1: $\mathrm{p}=0.007$, Hsp70: $\mathrm{p}=0.012$ ), confirming the negative impact of HSF1 haploinsufficiency in the expression of wellknown HSF1 targets (Fig. 3A-B).

A)

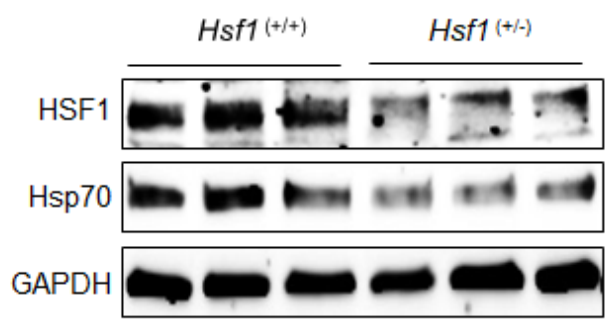

C)

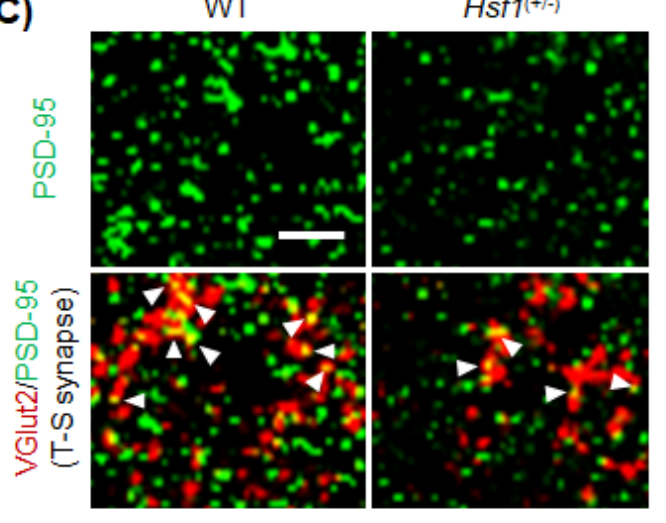

B)

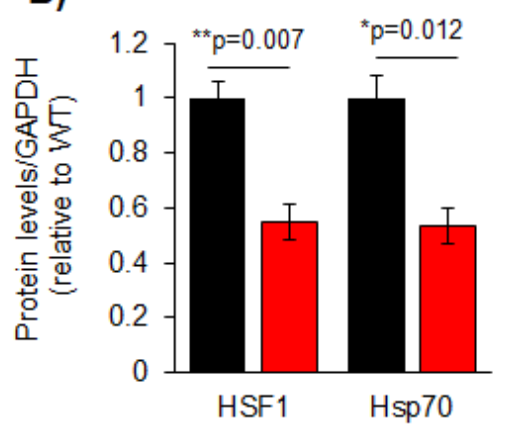

D)

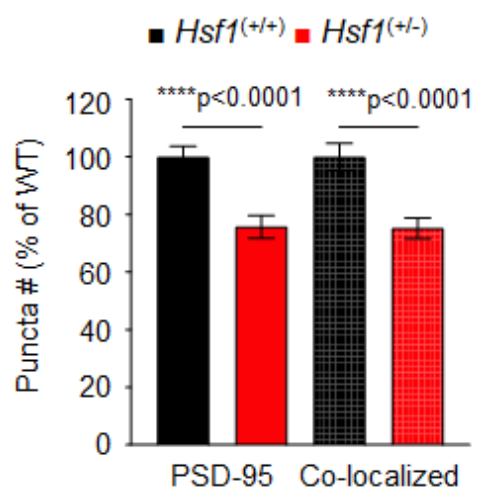

Figure 3. HSF1 haploinsufficiency decreased PSD-95 protein levels and striatal synapse density. A) Immunoblot from striatum samples of WT $\left(H s f 1^{(+/)}\right)$and $H s f 1^{(+/)}$mice at 6 months. GAPDH is used as a loading control. B) HSF1 and Hsp70 Protein levels quantified from A using ImageJ analyses. Data was normalized to GAPDH levels and relativized to WT. C) Immunohistochemistry for PSD-95 (green) and the pre-synaptic vesicular protein VGLUT2 (red) in the striatum of WT and $\mathrm{Hsfl}^{(+-)}$at 12 months. Arrows indicate co-localization between PSD-95 and VGLUT2. Scale bar 5 $\mu$ m. D) Puncta analysis for PSD-95 and co-localized PSD-95/VGlut2 from C. Punta number (\#) was relativized to WT. Error bars denote mean \pm SEM. N=3 mice/genotype. Unpaired Student's $t$ test. ${ }^{*} \mathrm{p}<0.05,{ }^{* *} \mathrm{p}<0.01,{ }^{* * *} \mathrm{p}<0.001,{ }^{* * * *} \mathrm{p}<0.0001$. Uncropped blots can be found in Supplementary Figure 3.

PSD-95 immunofluorescence in the dorsolateral striatum of $H s f 1^{(+-)}$mice revealed a significant reduction in the number of PSD-95 puncta (associated with synapses) 
compared to WT $(\mathrm{p}<0.0001)$ (Fig. 3C-D). In analyzing PSD-95 puncta by IF instead of total PSD-95 protein levels by immunoblotting, we were able to assess only the levels of synaptic PSD-95 in mature spines without considering non-synaptic, dendritic shaft levels of PSD-95 [40-42]. Previous studies in Hsf1(--) mice showed reduced PSD-95, aberrant synapse formation, impaired spinogenesis in the hippocampus, and working memory deficits $[14,17,18]$. Despite this evidence, this phenotype was associated with neurodevelopmental issues rather than a direct role of HSF1 in regulating PSD-95 and synapse stability.

Considering the role of PSD-95 in synapse formation and maintenance, we then assessed whether striatal synapse stability was affected in $H s f 1^{(+-)}$mice. Ex vivo analyses of the dorsolateral striatum using colocalization of VGlut2, a marker for thalamic presynaptic input, and PSD-95 were used to quantify thalamo-striatal excitatory synapse density, a major synaptic circuit involved in striatal function $[19,26,43,44]$. Levels of thalamo-striatal synapses were reduced in $H s f 1^{(+-)}$mice compared to WT $(\mathrm{p}<0.0001)$, paralleling the depletion seen in PSD-95 puncta (Fig. 3C-D), and indicating a worsening in synapse stability. Given these results as well as our previous in vitro studies, it can be concluded that modulating HSF1 levels leads to a depletion of PSD-95, an effect that can further lead to striatal synaptic dysfunction.

\subsection{HSF1 directly binds to HSEs present in the PSD-95 gene and regulates its transcription}

HSF1 regulates the expression of target genes by binding to HSEs, canonical sites that contain alternating inverted repeats of an nGAA sequence [11] (Fig. 4A). We investigated whether there were HSEs within the promoter or intergenic regions of Dlg4 that could explain a direct transcriptional regulation by HSF1. In silico analyses revealed four HSEs in the murine gene and one HSE in the human Dlg4 gene (Fig. 4B). Two HSEs (\#1 and \#2) were identified in the promoter region of the mouse PSD-95 gene and two HSEs (\#3 and \#4) were located within the coding DNA region (Fig. 4B). Interestingly, the only HSE identified in human Dlg4 (located in the promoter region) was identical to the murine HSE \#3 (cTTCctGAA). HSF1 chromatin immunoprecipitation (HSF1-ChIP) on all four murine Dlg4 HSEs in STHdh ${ }^{\mathrm{Q}^{7}}$ cells showed a significant enrichment compared to IgG (negative control) at HSE \#3, indicating HSF1 binds to this regulatory element in the murine Dlg4 gene (Fig. 4C). Therefore, we focused our subsequent HSF1-ChIP studies in brain samples on PSD-95 HSE \#3. Striatum samples were extracted from 3, 6, and 12 month WT animals to observe how HSF1 binding capacity to Dlg4 is altered over time (Fig. 4D). There were no significant differences in HSF1 binding to PSD-95 between 3 and 6 months although there was a significant reduction at 12 months $(\mathrm{p}=0.013)$, indicating a significant age effect on HSF1 binding capacity. The timing at which we observed a significant reduction in HSF1 binding on Dlg4 regulatory elements (between 6-12 months) preceded the reduction in levels of PSD-95 (between 12-22 months) (Fig. 1A-B).

To determine whether HSF1 directly regulated the expression of Dlg4 we conducted luciferase assays with a reporter containing the human PSD-95 promoter ( $\mathrm{h} P S D-95)$, which had HSE \#3 fused to luciferase (PSD95-pGL3a, obtained from Dr. Bao [8]), and we 
observed that luciferase activity was reduced after co-transfection with siHsf1. (Fig. 4E-F). We used site-directed mutagenesis to mutate the hPSD-95 HSE from GA->CT (Fig. 4G), thereby ablating HSF1 binding [45], to determine if direct regulation of Dlg4 expression by HSF1 is mediated through binding at HSE \#3. STHdh ${ }^{8}$ cells transfected with the mutated hPSD-95 HSE (Mut. HSE) showed a significant reduction in luciferase activity compared with WT hPSD-95 HSE (Fig. 4H). These results demonstrated HSF1 directly binds to Dlg4 regulatory elements and regulates its transcription, supporting the hypothesis that changes in levels of HSF1 in the mouse brain can directly lead to decreased expression of $D \lg 4$.

A)

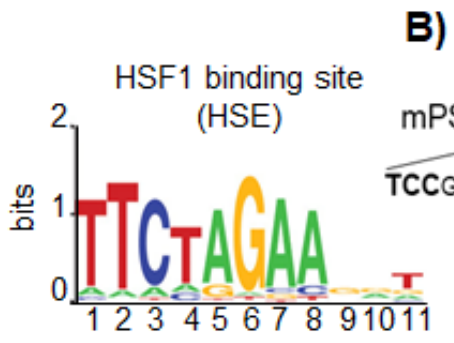

B)

MPSD-95

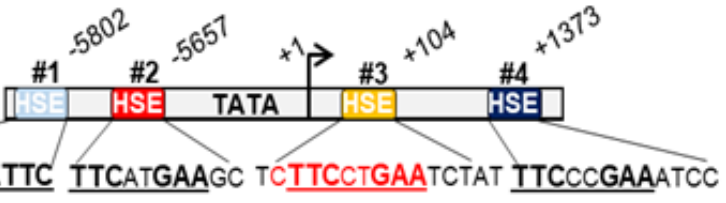

hPSD-95

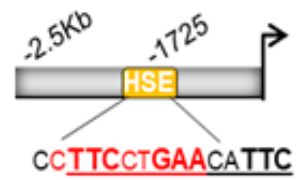

C)

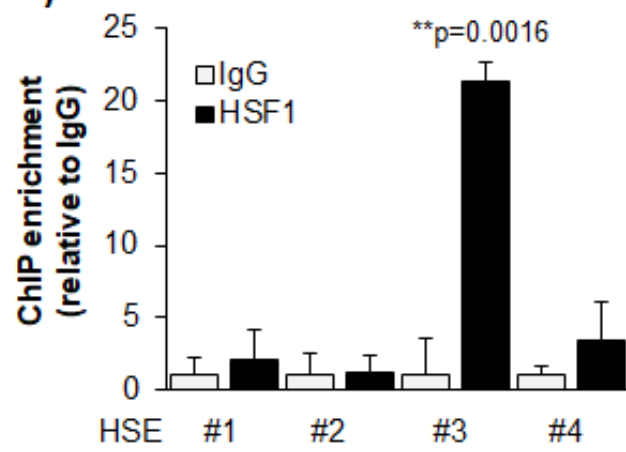

E)

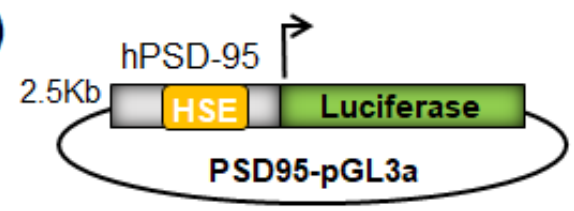

F)

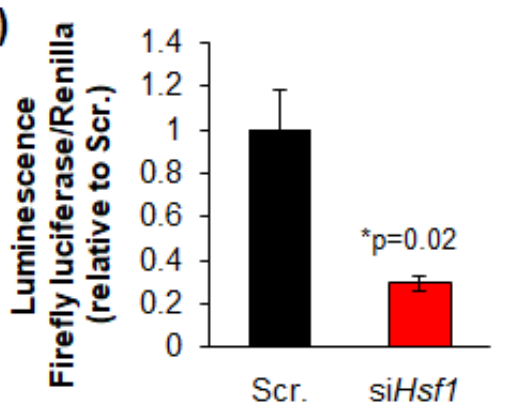

D)

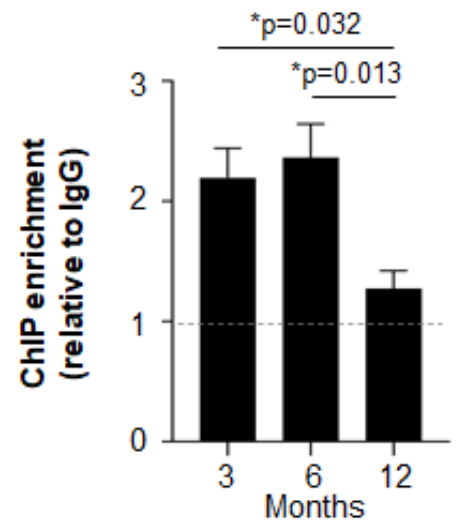

G)

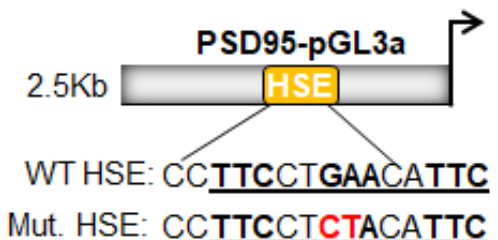

H)

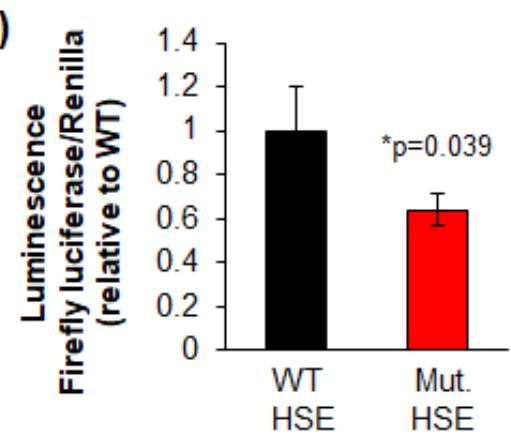

Figure 4. HSF1 binds to HSE within the mouse and human PSD-95 promoter/gene and directly regulates PSD-95 transcription. A) HSF1-binding motif (HSE) represented by MotifExpress. B) Genetic diagram of the HSEs found in the promoter and intergenic region of PSD-95 identified in silico. Mouse PSD-95 (mPSD-95) and human PSD-95 promoter (hPSD95) share an identical HSE (yellow). C) HSF1-ChIP for the four different HSEs identified in the mPSD-95 gene. Data is 
relative to IgG $(n=4)$. D) HSF1-ChIP for HSE \#3 in the striatum of WT mice at $3(n=4), 6(n=4)$ and 12 months (n=6). E) Diagram of PSD95-pGL3a vector expressing Luciferase under control of hPSD-95 promoter. F) Luciferase activity in $\mathrm{STH} d h^{\mathrm{Q} 7}$ cells transfected with non-targeting siRNA (Scr.) or siHSF1. Data is normalized to Renilla luciferase and relativized to Scr. G) Diagram of PSD95-pGL3a containing a WT HSE or a mutant HSE (Mut. HSE). H) Luciferase activity in STHdh ${ }^{\mathrm{Q7}}$ cells transfected with PSD95-pGL3a containing WT or Mut. HSE. Data is normalized to Renilla luciferase and relativized to WT. Error bars denote mean \pm SEM. Unpaired Student's t-test in C, F, H and One-way ANOVA with Tukey's post-hoc correction in D. ${ }^{*} \mathrm{p}<0.05,{ }^{* *} \mathrm{p}<0.01$.

\subsection{HSF1 binding to PSD-95 regulatory elements is impaired in HD}

Once established that HSF1 binds to and regulates Dlg4 transcription, we assessed whether this regulatory mechanism was altered in HD where both HSF1 protein levels and PSD-95 transcripts are reduced. HSF1-ChIP in STHdh ${ }^{\mathrm{Q} 111}$ cells compared to STHdh ${ }^{\mathrm{7}}$ revealed HSF1 binding to HSE \#3 in PSD-95 was significantly reduced ( $p=0.011$ ) (Fig. 5A). Additionally, HSF1-ChIP studies in zQ175 mice showed that at 3 months (presymptomatic) there are no differences in HSF1 binding to HSE \#3 in Dlg4 (Fig. 5B). However, at 6 months (symptomatic) HSF1 binding to HSE \#3 is reduced ( $\mathrm{p}=0.0409$ ) (Fig. 5C), coinciding with the onset of HSF1 depletion in this mouse model [19]. Although not statistically significant, HSF1-ChIP analyses in postmortem striatal tissue from patients with HD also revealed a trend $(\mathrm{p}=0.068)$ towards a reduction in HSF1 binding to human PSD-95 HSE \#3 compared to unaffected individuals (control) (Fig. 5D).
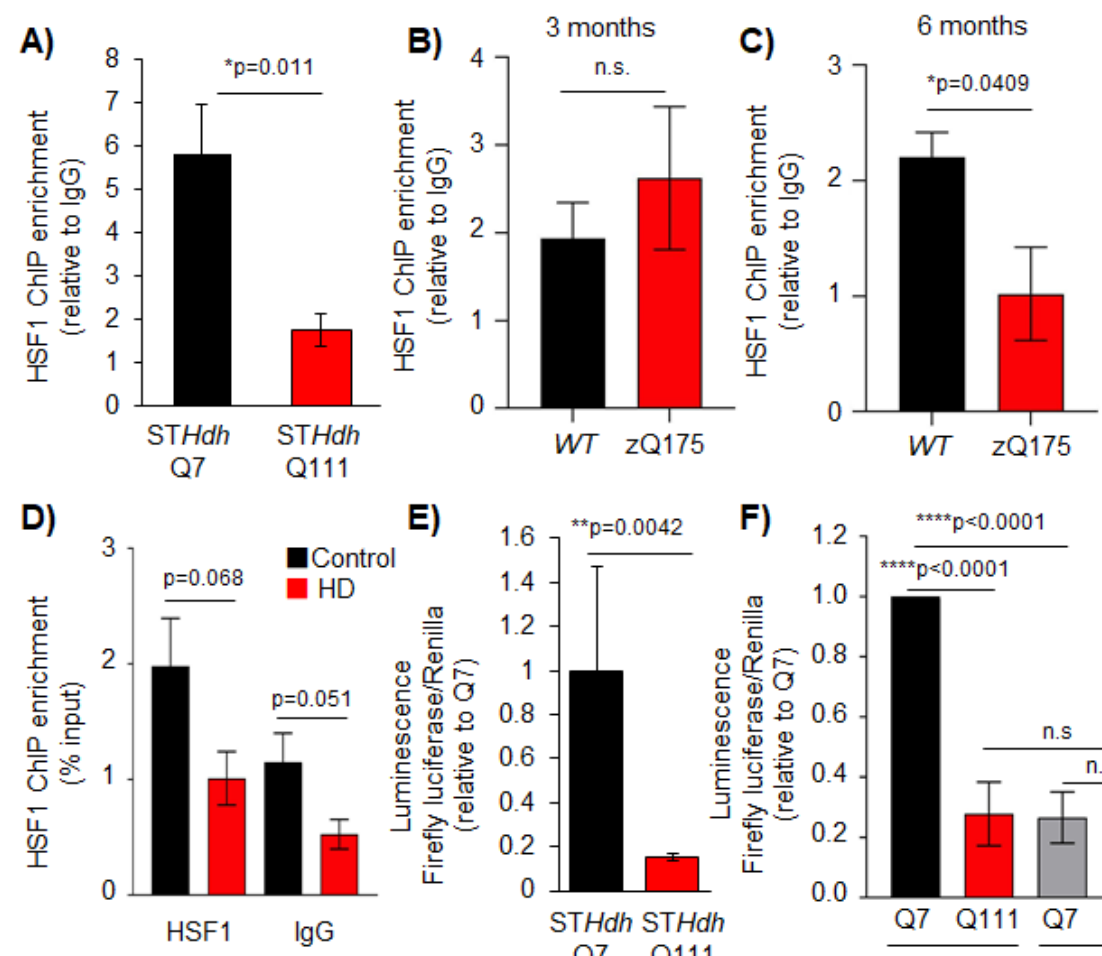

E)
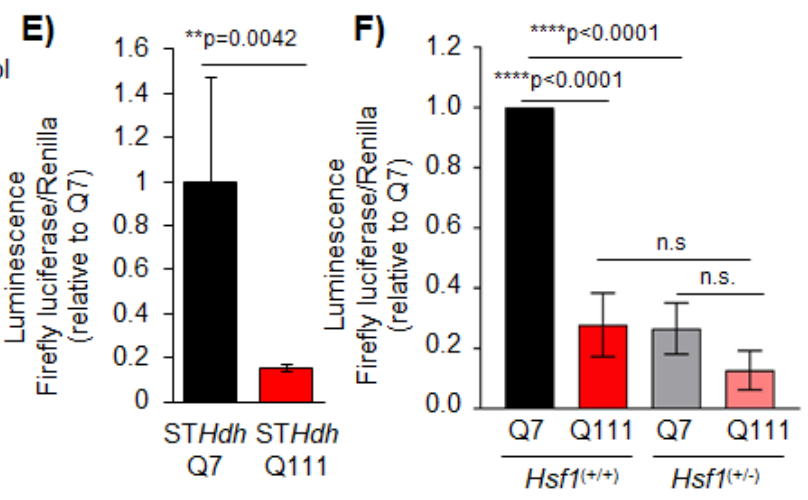

Figure 5. HSF1 binding to regulatory elements of PSD-95 and its regulation is impaired in HD. A-D) HSF1-ChIP on PSD-95 HSE \#3 in STHdh ${ }^{\mathrm{Q} 7}$ and STHdh ${ }^{\mathrm{Q} 111}$ cells $(\mathrm{n}=6)(\mathbf{A})$, in the striatum of WT and zQ175 mice at 3 (B) (n=4 mice/genotype) and 6 months $(\mathbf{C})(\mathrm{n}=4$ mice/genotype), and in the striatum of patients with HD and sex and age-matched controls (D) ( $\mathrm{n}=6$ individuals/group). E) Luciferase activity in $\mathrm{STH} d h^{\mathrm{Q} 7}$ and STHdh${ }^{\mathrm{Q} 111}$ cells. Data is normalized to Renilla luciferase and relativized to Q7 cells $(n=5)$. G) Diagram of PSD95-pGL3a containing a WT HSE or a mutant HSE (Mut. HSE). H) Luciferase activity in HSF1 (+/+) and HSF1 (+/-) STHdh ${ }^{\mathrm{Q} 7}$ and STHdh ${ }^{\mathrm{Q} 111}$ cells. Data is normalized to Renilla luciferase and relativized to Q7:HSF1(+/+). Error bars denote mean \pm SEM. Unpaired Student's t-test in A-E, and One-way ANOVA with Tukey's post-hoc correction in F. ${ }^{*} \mathrm{p}<0.05,{ }^{* *} \mathrm{p}<0.01,{ }^{* * *} \mathrm{p}<0.001,{ }^{* * * *} \mathrm{p}<0.0001$. 
We conducted luciferase assays in STHdh ${ }^{\mathrm{Q} 7}$ and STHdhQ111 cells using the PSD95pGL3a plasmid. The results obtained revealed a significant decrease in luciferase activity in HD cells relative to control cells $(\mathrm{p}=0.0042)$ (Fig. 5E). Furthermore, transfection of

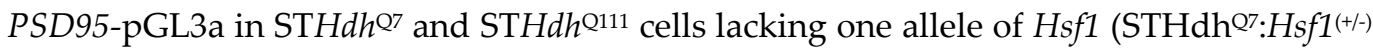
and STHdhQ111:Hsf1(+/)) showed a significant reduction $(\sim 90 \%, \mathrm{p}<0.0001)$ in luciferase activity in both cell lines when HSF1 levels were reduced (Fig. 5F). Taken together our data indicated the pathological depletion of HSF1 in HD results in decreased HSF1 binding to PSD-95 and subsequent decreased expression of this synaptic protein.

\section{Discussion}

Age-related cognitive decline as well as motor and cognitive impairments seen in HD and other neurodegenerative diseases are influenced by progressive synaptic dysfunction. Transcriptional dysregulation and alterations in Dlg4 levels have proven to influence synaptic function and stability. Dlg4 RNAi knockdown or genetic deletion $\left(D l g 4^{\mathrm{KO}}\right)$ resulted in decreased glutamate-dependent AMPAR clustering and transmission, both important for long-term synaptic plasticity and memory [2], while overexpression of PSD-95 in hippocampal neurons produced the opposite effect [1]. Downregulation of PSD-95 has been shown in various brain regions during aging, $A D$, and in the striatum of mice and patients with HD, but the mechanism responsible for such dysregulation is unknown $[3-6,25,36,46]$. In this study we have demonstrated that the stress protective transcription factor HSF1, known for its role in the regulation of protein quality control machinery and progressively depleted in aging and HD, contributes to the transcriptional regulation of Dlg4. These findings confirmed a regulatory role for HSF1 that has meaningful implications for synapse stability in aging and neurodegeneration.

The involvement of HSF1 in synapse regulation was previously associated with its role in regulating protein quality control systems and the expression of chaperones. These proteins are required for newly synthesized synaptic protein folding under both basal and stress conditions to ensure synaptic protein homeostasis, reorganization of the synaptic actin cytoskeleton, and modulation of signaling cascades involved in memory formation [15]. This is supported by the presence of several chaperones like HSP90, HSP60, and HSC70 in synaptosomes isolated from rat forebrain and cerebellum, indicating their involvement in basal synaptic function [16]. In addition, HSP70, another target of HSF1, was enriched in rat synaptosomes, particularly at the PSD, following hypothermiainduced stress, demonstrating the necessity of chaperones in maintaining synaptic homeostasis under stress conditions [16]. It has been widely demonstrated that chaperone levels decrease in aging and HD, which is influenced by the abnormal post-translational degradation of HSF1 under these conditions[11,19,47]. Therefore, it is reasonable to hypothesize that HSF1 could control synaptic function and stability by regulating the levels of chaperones, and its degradation could impact the concentration and composition of the chaperone pool at synapses thereby altering synaptic function. However, further studies are required to directly establish this regulatory mechanism. 
Other studies have suggested a more direct role of HSF1 in the regulation of synaptic function by controlling synaptic components. Studies using Hsf1 ${ }^{\mathrm{KO}}$ mice showed reduced PSA-NCAM (polysialylated-neural cell adhesion molecule), known to participate in the remodeling of neuronal circuits, and reduced hippocampal spine density [17]. In this study the authors also reported reduced PSD-95 protein levels in the hippocampus of $H s f 1^{\mathrm{KO}}$ mice, which was reversed by the overexpression of a constitutively active form of HSF1 (caHSF1) in neonatal Hsf1 КО mice [17]. While the study did not assess how HSF1 participated in the regulation of PSD-95, it highlighted a potential connection between the levels of HSF1 and PSD-95. In addition, AD mice or primary hippocampal neurons treated with 17-AAG, an HSP90 inhibitor that leads to activation of HSF1, resulted in increased expression of PSD-95 as well as the pre-synaptic proteins Synapsin I and Synaptophysin and BDNF (brain-derived neurotrophic factor) [5]. However, it was unclear whether the effects on the expression of these various synaptic components by 17-AAG were HSF1dependent or if HSF1 was directly involved in their regulation. We have now shown that manipulating levels of HSF1 in vitro as well as analyse in $H s f 1^{(+/-)}$mice leads to decreased expression and protein levels of PSD-95. We showed that this effect is mediated by the direct binding of HSF1 onto an HSE within the Dlg4 gene/promoter in both mice and humans. It was corroborated following the luciferase experiments that HSF1 directly regulates the expression of Dlg4 in cells and mice. Similarly, Ting and colleagues [48] showed that HSF1 directly binds to HSEs in the promoter of SAP97, another MAGUK family synaptic scaffolding protein with various functions in the regulation of synaptic receptor clustering, and regulated SAP97 expression in cardiomyocytes. These data confirm the direct role of HSF1 in regulating PSD-95 and other synaptic genes.

HSF1 binding to Dlg4 regulatory elements decreased during aging which paralleled the depletion of HSF1 and PSD-95 protein levels. However, we observed that HSF1 protein levels started to decrease between 3-6 months of age while HSF1 binding capacity to Dlg4 was significantly reduced between 6-12 months; PSD-95 protein was not significantly reduced until 12-22 months. This could indicate that depletion of HSF1 does not immediately translate to decreased binding. It is possible that HSF1 maintains its regulatory functions on some genes up to a certain threshold. Once HSF1 is reduced below a minimum functional concentration, the consequences of HSF1 depletion on DNA binding are more evident. While we have shown a direct regulatory role of HSF1 on Dlg4 expression, decreased HSF1 binding in vivo does not immediately translate into the reduction of PSD-95 protein levels. One possibility is that other unknown transcription factors, in conjunction with HSF1, contribute to maintaining some basal PSD-95 expression. Alternatively, the stabilization of PSD-95 protein can be regulated by posttranslational events [49] even when the mRNA levels are depleted, explaining how the protein is maintained for a period of time before its eventual depletion.

We showed that age-dependent depletion of HSF1 binding on the Dlg4 gene is exacerbated in the presence of mtHTT in both cell and mouse models, which correlates with the pathological depletion of HSF1 and PSD-95 reported in HD models and patients with HD. Consistent with our findings, previous ChIP-seq analyses in STHdhQ7 and 
STHdhQ111 cells showed that mtHTT dramatically alters the genome-wide binding of HSF1 [50]. In particular, the HSF1 gene targets most affected by the presence of mtHTT were genes associated with cytoskeletal binding, focal adhesion, and GTPase activity, several of which have synaptic functions [50]. HSF1 is abnormally degraded in HD due to phosphorylation-dependent proteasomal degradation mediated by Protein Kinase CK2 $\alpha^{\prime}$ [19]. CK2 $\alpha^{\prime}$ haploinsufficiency in the zQ175 HD mouse model (zQ175:CK2 $\alpha^{\prime(+-))}$ ) rescued levels of HSF1 in the striatum and increased Dlg4 expression compared to zQ175 mice, in addition to ameliorating many HD-like phenotypes [51]. Interestingly, RNA-seq studies performed in the striatum of $\mathrm{zQ} 175$ and $\mathrm{zQ} 175: \mathrm{CK} 2 \alpha^{\prime(+)-)}$ revealed that signaling pathways related to synaptogenesis and glutamate receptor signaling, which are significantly dysregulated in zQ175, were specifically rescued in zQ175:CK2 $\alpha^{\prime(+-)}$ mice. Taken together, these data suggest impaired HSF1 binding in HD largely has effects not only on PSD-95 regulation but also on other synaptic pathways. However further studies are needed to directly assess the association between synaptic transcriptional changes in HD and alterations in HSF1 binding.

An important aspect of our study is the intersection between HSF1 regulatory function, $D \lg 4$ expression, and their connection with excitatory synapse density. Our data showed $H s f 1^{(+-)}$mice, which present decreased PSD-95 levels, resulted in a loss of thalamostriatal (T-S) excitatory synapses, an important synaptic circuit involved in cognitive functions such as goal-directed learning, action selection, and flexible control of behavior, all of which are disrupted in HD [43,44,52-54]. Excitatory synapse impairment is a major pathological hallmark in HD [55,56]. We previously showed thalamo-striatal excitatory synapse density is reduced as early as 5 weeks in zQ175 mice [19], which coincided with the reduction in PSD-95 puncta observed at this time. The resemblance between $\mathrm{HSF}^{(+-)}$ and zQ175 regarding PSD-95 dysregulation and loss of T-S synapses further demonstrates the involvement of HSF1 in controlling excitatory synapse stability in the striatum. Depletion of T-S synapses precedes mtHTT aggregation and symptom onset in zQ175 and is believed to initiate striatal pathology. Therefore, any therapeutic manipulations aimed at preventing T-S synapse loss can result in long-term benefits. We have recently shown that increased levels of HSF1 in the $\mathrm{zQ} 175: \mathrm{CK} 2 \alpha^{\prime(+-)}$ mouse resulted in a rescue of T-S synapses sustained up to 12 months of age [19], which correlated with improved motor coordination. In addition, this model increased the frequency of AMPA-mediated miniature excitatory postsynaptic currents, which reflects the probability of neurotransmitter release from presynaptic vesicles and also correlates with the number of synapses [51]. Overall, these data provide a strong connection between HSF1, PSD-95 and the regulation of T-S synapse density. However, the dependency of HSF1 on the effects mediated by $\mathrm{CK} 2 \alpha^{\prime}$ haploinsufficiency still needs to be investigated.

In summary, our study has provided evidence confirming the regulatory role of HSF1 in the direct regulation of synaptic components, such as PSD-95, and their connection with excitatory synaptic maintenance. Future analyses will be needed to assess the impact of HSF1 in the regulation of other synaptic genes whose expression is dysregulated 
in the context of aging and neurodegeneration, and their overall contribution to the pathological dysfunction in various synaptic circuits.

\section{Materials and Methods}

\subsection{Cell Lines}

Mammalian cell lines used in this study were the mouse-derived striatal cells

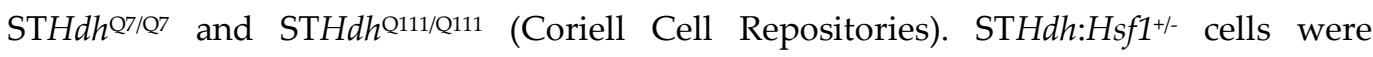
generated using pSpCas9-(BB)-2A-GFP (PX458, Addgene \#48138) [57] and a gRNA targeting HSF1- exon9 (5'caccGAGTACCCGAGGGCTGTGAGGCTCATGGGCTCCCGACACTCCcaaa-3').

Efficacy of indel generation was tested using the SURVEYOR ${ }^{\mathrm{TM}}$ nuclease assay. Transfected cells were sorted by FACS and individual GFP+ cells were plated into 96 well plates. Colonies from isolated clones were genotyped using HSF1 primers (Forward: 5' CCTTAGTGGGTCAGCCTTTATG-3', Reverse: 5'-AGGGGCATATCCCATTTCTAGT-3').

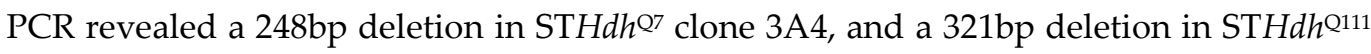
clone 8E6. Cells were grown at $33^{\circ} \mathrm{C}$ in Dulbecco's modified Eagle's medium (DMEM, Genesee) supplemented with $10 \%$ fetal bovine serum (FBS), $100 \quad \mathrm{U} \mathrm{ml}^{-1}$ penicillin/streptomycin and $100 \mu \mathrm{g} \mathrm{ml}^{-1} \mathrm{G} 418$ (Gibco), as previously described (RGP, 2017).

\subsection{Mouse Strains}

For this study we used a full-length knock-in mouse model of HD known as zQ175 on the C57BL/6J background (Stock No. 027410). Sperm from HSF1 heterozygous knock-

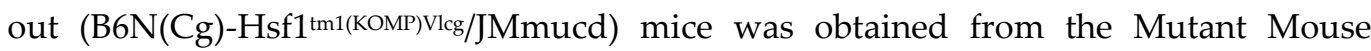
Resource and Research Center (University of California, Davis) (Stock No. 048101-UCD) and generated by the Knockout Mouse Phenotyping Program (KOMP2). In vitro fertilization using C57BL/6N females was conducted at the Mouse Genetics laboratory at University of Minnesota. Hsf1+l: $\mathrm{m} 11$ mice were crossbred with CMV-CRE mice (B6.C$\mathrm{Tg}(\mathrm{CMV}-\mathrm{cre}) 1 \mathrm{Cgn} / \mathrm{J}$ ( (Stock No. 006054) to delete the Neomycin cassette flanked by loxP

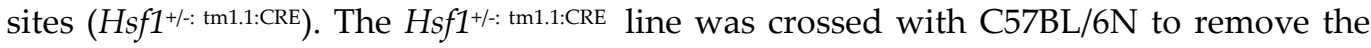
CRE gene ( $H s f 1^{+/-: t m 1.1}$, referenced as $H s f 1^{(+-)}$in this study). All animal care and sacrifice procedures were approved by the University of Minnesota Institutional Animal Care and Use Committee (IACUC) in compliance with the National Institutes of Health guidelines for the care and use of laboratory animals under the approved animal protocol 200738316A.

\subsection{Human Samples}

HD brain tissues were obtained from Harvard Brain Tissue Resource Center. Cases with and without clinical neurological disease were processed in the same way following the same sampling protocols. Control and HD cases were compared pairwise for sex and age.

\subsection{Immunoblot Analysis}


Sample preparation and immunoblotting conditions were performed as previously described [19]. Cell and striatum protein extracts from one hemisphere of mice were prepared in cell lysis buffer ( $25 \mathrm{mM}$ Tris $\mathrm{pH} 7.4,150 \mathrm{mM} \mathrm{NaCl}, 1 \mathrm{mM}$ EDTA, $1 \%$ TritonX100 and $0.1 \%$ SDS) supplemented with phosphatase and protease inhibitors (Thermo Scientific). Protein samples were separated on 4-20\% SDS Criterion TGX Stain-Free gels (BioRad) and transferred to a nitrocellulose membrane (BioRad $0.2 \mu \mathrm{m}$ ). Primary antibodies used are as follows: anti-GAPDH (Santa Cruz, sc-365062,1:10000), anti-HSF1 (Bethyl, A303-176A, 1:1000), anti-PSD-95 (Novus, NB300-556, 1:1000), and anti-Hsp70 (Enzo, C92F3A-5, 1:1000 ). Quantitative analyses were performed using ImageJ software and normalized to GAPDH controls.

\subsection{Immunohistochemistry $\&$ synapse density analyses}

Sample preparation was performed as previously described [19]. Fluorescent images from dorsal striatum (bregma 0.5-1.1 mm) were acquired on a confocal microscope (Olympus FV1000). Primary antibodies used are as follows: VGLUT2 (Millipore AB2251I, 1:1000), PSD-95 (Thermofisher 51-6900, 1:500) . Secondary antibodies used are as follows: goat anti-guinea pig Alexa 488 (1:200) and goat anti-rabbit Alexa 594 (1:200) (Invitrogen). Confocal scans (optical section depth $0.34 \mathrm{~mm}, 15$ sections per scan) in the dorsal striatum were performed at 60X magnification. Maximum projections of three consecutive optical sections were generated. Puncta analyses were conducted blinded using the PunctaAnalyzer Plugin on ImageJ, as previously described $[19,26]$.

\section{6. $R N A$ preparation and $R T-q P C R$}

RNA was extracted from STHdh cells and mouse striatal tissues using the RNeasy extraction kit (Qiagen) according to the manufacturer's instructions. cDNA was prepared using the Superscript First Strand Synthesis System (Invitrogen) according to the manufacturer's instructions. SYBR green based qPCR was performed with SYBR mix (Roche \& Genesee) using the LightCycler 480 System (Roche). Primers used are as follows, GAPDH (Forward: 5'ACACATTGGGGGTAGGAACA-3', Reverse: 5'AACTTTGGCATTGTGGAAGG-3'), PSD-95 (Forward: 5' CCGCGATTACCACTTTGTCT-3', Reverse: 5'-ACGGATGAAGATGGCGATAG-3'). Each sample was tested in triplicate and normalized to GAPDH levels. For analysis, the ${ }^{\Delta \Delta} \mathrm{Ct}$ method was used to calculate the relative fold gene expression.

\section{7. siRNA transfection}

For HSF1 knock-down, STHdh cells were transfected at 75\% confluency with a FlexiTube siRNA solution GS15499 (10 $\mu$ mol from Qiagen) using DharmaFECT1 transfection reagent as per manufacturer's instructions. As a negative control, nontargeting siRNA was used. Cells were incubated at $33^{\circ} \mathrm{C}$ for $24 \mathrm{~h}$. followed by RNA extraction and RT-qPCR. All siRNAs have been validated by RT-qPCR and immunoblotting for knockdown efficiency. 


\subsection{Chromatin Immunoprecipitation}

Chromatin immunoprecipitation in cell lines was performed as previously described [19]. Cells were grown to $75 \%$ confluency, placed on ice and cross-linked with $37 \%$ Formaldehyde followed by glycine quenching $(125 \mathrm{mM})$. Chromatin shearing was performed using 3 sets of 40 s sonication at 20\% amplitude. In the case of mouse tissue, 15 mg of frozen striatal tissue was crosslinked and quenched, as described for cell lines, and sonication was performed using 3 sets of 20 second pulses at $40 \%$ amplitude. In the case of human tissue, $\sim 75 \mathrm{mg}$ frozen tissue from caudate/putamen of postmortem samples obtained from Harvard biobank were crosslinked and quenched, as described for cell lines. Sonication of human tissue was performed using 3 sets of 20 second pulses at 50\% amplitude. Input samples were saved prior to addition of antibodies. $2 \mu \mathrm{g}$ of antibody (rabbit HSF1: Bethyl A303-176A and rabbit IgG: R\&D Systems AF008) was added to cell and mouse samples and $4 \mu \mathrm{g}$ antibody was used for human samples. Samples were incubated overnight at $4^{\circ} \mathrm{C}$. For cell lines immunoprecipitation (IP) was performed using Protein G agarose beads (Invitrogen, 15-920-010) and Dynabeads ${ }^{\mathrm{TM}}$ Protein G (Invitrogen, 10009D) for mice and humans, followed by chromatin purification using the Qiaquick min-elute PCR purification kit (Qiagen) per manufacturer's instructions. SYBR green (Roche and Genesee) qPCR was performed on IP and input samples. ${ }^{\Delta} \mathrm{Ct}$ method was used to determine relative amounts of DNA normalized to input. Binding of HSF1 was evaluated using primers spanning the four different HSE on the murine and human PSD95 promoter/gene (HSE 1\#; Forward:5'-GAGCCACAAACAGTCGAA-3', Reverse: 5'TGGAAAGTGGCAGATGAGTG-3'; HSE \#2: Forward: 5'-CCCACCTCTCCTAGCACAT3', Reverse: 5'-ATCATGAGACCC-3'; HSE \#3: Forward: 5'GGTCTTTGAGGGGGTGATCT-3', Reverse: 5'-CTGACCTGGGAGCTGGTAAA-3'; HSE \#4: Forward: 5'-TCTCCTCCTCTCTCCССТTC-3', Reverse: 5'CACACCCCGATTCTCAGG-3'); human HSE: Forward: 5' TCACTGCCCCTCCCTTAGTA-3', Reverse: 5'-GGGGTTTTACGGGTAAGAGG-3') and values were normalized against IgG.

\subsection{Luciferase assays}

We used the Dual-Luciferase ${ }^{\circledR}$ Reporter (DLR ${ }^{\mathrm{TM}}$ ) Assay (Promega, E1910) per manufacturer's instructions. We used PSD95-pGL3a (a generous gift from Dr. Bao, Washington University) [8] and substituted the firefly luciferase gene with the pGL4 derived firefly. Mutant HSE PSD95-pGL3a was generated by site-directed mutagenesis to substitute GA $\rightarrow$ CT within the HSE. $2 \mu \mathrm{g}$ of modified PSD95-pGL3a or mut. HSE PSD95pGL3 and $2 \mu \mathrm{g}$ of Renilla luciferase vector (control reporter) were co-transfected into STHdh cells and incubated for $12 \mathrm{~h}$. Cells were then harvested and plated into a 96 well plate to measure luciferase activity. Luciferase activity (luminescence) was calculated by dividing the signal from Firefly and Renilla luciferase and was relativized to the control sample.

Supplementary Materials: The following are available online at www.mdpi.com/xxx/s1, Figure S1S3: Uncropped immunoblots from Figure 1-3. 
Author Contributions: Conceptualization, R.G.P; methodology, R.G.P, N.Z., J.S., D.Y., W.T., M.S. and L.C.; formal analysis, R.G.P, N.Z., J.S., W.T.; investigation, R.G.P, N.Z., J.S., W.T.; writingoriginal draft preparation, N.Z.; writing - review and editing, R.G.P., N.Z., J.S.; funding acquisition, R.G.P. All authors have read and agreed to the published version of the manuscript."

Funding: This research was funded by the National Institute of Neurological Disorders and Stroke (R01NS110694) to R.G.P. and the Biomedical Research Awards for Interdisciplinary New Science (BRAINS) University of Minnesota to R.G.P.

Institutional Review Board Statement: All animals work was conducted according to procedures approved by the University of Minnesota Institutional Animal Care and Use Committee (IACUC) in compliance with the National Institute of Health guidelines for the care are use of laboratory animals under the approved animal protocol 200-38316A. Work with human postmortem specimens was waived by the University of Minnesota Institutional Review Board (IRB). The IRB determined that the proposed activity is not research involving human subjects as defined by DHHS and FDA regulations. To arrive at this determination, the IRB used "WORKSHEET: Human Research (HRP-310)."

Data Availability Statement: All data generated in this study in presented in the current manuscript. No new datasets were generated. Data is available upon request from the corresponding author.

Conflicts of Interest: The Authors declare no conflict of interest.

\section{References}

1. El-Husseini, A.; Schnell, E.; Chetkovich, D.; Nicoll, R.; Bredt, D. PSD-95 involvement in maturation of excitatory synapses. Science 2000, 290, 1364-8.

2. Béïque, J.-C.; Lin, D.-T.; Kang, M.-G.; Aizawa, H.; Takamiya, K.; Huganir, R.L. Synapse-specific regulation of AMPA receptor function by PSD-95. Proc. Natl. Acad. Sci. 2006, 103, 19535-19540.

3. Li, Y.; Abdourahman, A.; Tamm, J.; Pehrson, A.; Sánchez, C.; Gulinello, M. Reversal of age-associated cognitive deficits is accompanied by increased plasticity-related gene expression after chronic antidepressant administration in middle-aged mice. Pharmacol. Biochem. Behav. 2015, 135, 70-82.

4. Carretón, O.; Giralt, A.; Torres-Peraza, J.; Brito, V.; Lucas, J.; Ginés, S.; Canals, J.; Alberch, J. Age-dependent decline of motor neocortex but not hippocampal performance in heterozygous BDNF mice correlates with a decrease of cortical PSD-95 but an increase of hippocampal TrkB levels. Exp. Neurol. 2012, 237, 335-345.

5. Chen, Y.; Wang, B.; Liu, D.; Li, J.J.; Xue, Y.; Sakata, K.; Zhu, L.Q.; Heldt, S.A.; Xu, H.; Liao, F.F. Hsp90 chaperone inhibitor 17AAG Attenuates A $\beta$-induced synaptic toxicity and memory impairment. J. Neurosci. 2014, 34, 2464-2470.

6. Hodges, A.; Strand, A.; Aragaki, A.; Kuhn, A.; Sengstag, T.; Hughes, G.; Elliston, L.; Hartog, C.; Goldstein, D.; Thu, D.; et al. Regional and cellular gene expression changes in human Huntington's disease brain. Hum. Mol. Genet. 2006, 15, 965-977.

7. Qin, X.; Jiang, Y.; Tse, Y.; Wang, Y.; Wong, T.; Paudel, H. Early Growth Response 1 (Egr-1) Regulates N-Methyl-d-aspartate Receptor (NMDAR)-dependent Transcription of PSD-95 and $\alpha$-Amino-3-hydroxy-5-methyl-4-isoxazole Propionic Acid Receptor (AMPAR) Trafficking in Hippocampal Primary Neurons. J. Biol. Chem. 2015, 290, 29603-29616.

8. Bao, J.; Lin, H.; Ouyang, Y.; Lei, D.; Osman, A.; Kim, T.-W.; Mei, L.; Dai, P.; Ohlemiller, K.K.; Ambron, R.T. Activitydependent transcription regulation of PSD-95 by neuregulin-1 and Eos. Nat. Neurosci. 2004711 2004, 7, 1250-1258.

9. Zheng, S.; Gray, E.E.; Chawla, G.; Porse, B.T.; O’Dell, T.J.; Black, D.L. PSD-95 is post-transcriptionally repressed during early neural development by PTBP1 and PTBP2. Nat. Neurosci. 2012153 2012, 15, 381-388.

10. Willett, C.E.; Kawasaki, H.; Amemiya, C.T.; Lin, S.; Steiner, L.A. Ikaros expression as a marker for lymphoid progenitors during zebrafish development. Dev. Dyn. 2001, 222, 694-698.

11. Gomez-Pastor, R.; Burchfiel, E.T.; Thiele, D.J. Regulation of heat shock transcription factors and their roles in physiology and disease. Nat. Publ. Gr. 2017, 19, 4-19. 
shock factor 1 (HSF1) converging mechanisms. Neural Regen. Res. 2020, 15, 69-70.

13. Hooper, P.L.; Durham, H.D.; Török, Z.; Hooper, P.L.; Crul, T.; Vígh, L. The central role of heat shock factor 1 in synaptic fidelity and memory consolidation. Cell Stress Chaperones 2016, 21, 745-753.

14. Homma, S.; Jin, X.; Wang, G.; Tu, N.; Min, J.; Yanasak, N.; Mivechi, N. Demyelination, astrogliosis, and accumulation of ubiquitinated proteins, hallmarks of CNS disease in hsf1-deficient mice. J. Neurosci. 2007, 27, 7974-7986.

15. Gorenberg, E.; Chandra, S. The Role of Co-chaperones in Synaptic Proteostasis and Neurodegenerative Disease. Front. Neurosci. 2017, 11.

16. Bechtold, D.A.; Rush, S.J.; Brown, I.R. Localization of the Heat-Shock Protein Hsp70 to the Synapse Following Hyperthermic Stress in the Brain. J. Neurochem. 2000, 74, 641-646.

17. Uchida, S.; Hara, K.; Kobayashi, A.; Fujimoto, M.; Otsuki, K.; Yamagata, H.; Hobara, T.; Abe, N.; Higuchi, F.; Shibata, T.; et al. Impaired hippocampal spinogenesis and neurogenesis and altered affective behavior in mice lacking heat shock factor 1 . Proc. Natl. Acad. Sci. 2011, 108, 1681-1686.

18. Zhu, X.; Cheng, M.; Peng, M.; Xiao, X.; Yao, S.; Zhang, X. Basal behavioral characterization of hsf1 deficient mice and its cellular and behavioral abnormalities underlying chronic unpredictable stressors. Behav. Brain Res. 2008, 193, $225-229$.

19. Gomez-Pastor, R.; Burchfiel, E.T.; Neef, D.W.; Jaeger, A.M.; Cabiscol, E.; Mckinstry, S.U.; Doss, A.; Aballay, A.; Lo, D.C.; Akimov, S.S.; et al. Abnormal degradation of the neuronal stress-protective transcription factor HSF1 in Huntington's disease. Nat. Commun. 2017, 8.

20. Goetzl, E.J.; Boxer, A.; Schwartz, J.B.; Abner, E.L.; Petersen, R.C.; Miller, B.L.; Carlson, O.D.; Mustapic, M.; Kapogiannis, D. Low neural exosomal levels of cellular survival factors in Alzheimer's disease. Ann. Clin. Transl. Neurol. $2015,2,769$.

21. Chafekar, S.M.; Duennwald, M.L. Impaired Heat Shock Response in Cells Expressing Full-Length Polyglutamine-Expanded Huntingtin. PLoS One 2012, 7, e37929.

22. Maheshwari, M.; Bhutani, S.; Das, A.; Mukherjee, R.; Sharma, A.; Kino, Y.; Nukina, N.; Ranjan Jana, N. Dexametha sone induces heat shock response and slows down disease progression in mouse and fly models of Huntington's disease. Hum. Mol. Genet. 2014, 23, 2737-2751.

23. Kim, E.; Wang, B.; Sastry, N.; Masliah, E.; Nelson, P.; Cai, H.; Liao, F. NEDD4-mediated HSF1 degradation underlies $\alpha$ synucleinopathy. Hum. Mol. Genet. 2016, 25, 211-222.

24. MacDonald, M.E.; Ambrose, C.M.; Duyao, M.P.; Myers, R.H.; Lin, C.; Srinidhi, L.; Barnes, G.; Taylor, S.A.; James, M.; Groot, N.; et al. A novel gene containing a trinucleotide repeat that is expanded and unstable on Huntington's disease chromosomes. Cell 1993, 72, 971-983.

25. Peng, Q.; Wu, B.; Jiang, M.; Jin, J.; Hou, Z.; Zheng, J.; Zhang, J.; Duan, W. Characterization of Behavioral, Neuropathological, Brain Metabolic and Key Molecular Changes in zQ175 Knock-In Mouse Model of Huntington's Disease. PLoS One 2016, 11, $\mathrm{e} 0148839$

26. Mckinstry, S.U.; Karadeniz, Y.B.; Worthington, A.K.; Hayrapetyan, V.Y.; Ozlu, M.I.; Serafin-Molina, K.; Risher, X.W.C.; Ustunkaya, T.; Dragatsis, I.; Zeitlin, X.S.; et al. Huntingtin Is Required for Normal Excitatory Synapse Development in Cortical and Striatal Circuits. Neurobiol. Dis. 2014, 34, 9455-9472.

27. Rieckmann, A.; Johnson, K.A.; Sperling, R.A.; Buckner, R.L.; Hedden, T. Dedifferentiation of caudate functional connectivity and striatal dopamine transporter density predict memory change in normal aging. Proc. Natl. Acad. Sci. 2018, 115, 1016010165 .

28. Lighthall, N.R.; Pearson, J.M.; Huettel, S.A.; Cabeza, R. Feedback-Based Learning in Aging: Contributions and Trajectories of Change in Striatal and Hippocampal Systems. J. Neurosci. 2018, 38, 8453-8462.

29. Ou, X.; Buckwalter, G.; McNeill, T.H.; Walsh, J.P. Age-related change in short-term synaptic plasticity intrinsic to excitatory striatal synapses. Synapse 1997, 27, 57-68. 
30. Menalled, L.B.; Kudwa, A.E.; Miller, S.; Fitzpatrick, J.; Watson-Johnson, J.; Keating, N.; Ruiz, M.; Mushlin, R.; Alosio, W.; McConnell, K.; et al. Comprehensive Behavioral and Molecular Characterization of a New Knock-In Mouse Model of Huntington's Disease: zQ175. PLoS One 2012, 7, e49838.

31. Vonsattel, J.; Myers, R.; Stevens, T.; Ferrante, R.; Bird, E.; Richardson, E. Neuropathological classification of Huntington's disease. J. Neuropathol. Exp. Neurol. 1985, 44, 559-577.

32. Georgiou-Karistianis, N.; Stout, J.C.; D., J.F.D.; Carron, S.P.; Ando, A.; Churchyard, A.; Chua, P.; Bohanna, I.; Dymowski, A.R.; Poudel, G.; et al. Functional magnetic resonance imaging of working memory in Huntington's disease: Cross-sectional data from the IMAGE-HD study. Hum. Brain Mapp. 2014, 35, 1847-1864.

33. Henley, J.; Wilkinson, K. AMPA receptor trafficking and the mechanisms underlying synaptic plasticity and cognitive aging. Dialogues Clin. Neurosci. 2013, 15, 11-27.

34. Morigaki, R.; Goto, S. Postsynaptic Density Protein 95 in the Striosome and Matrix Compartments of the Human Neostriatum. Front. Neuroanat. 2015, 0, 154.

35. Jurivich, D.; Manocha, G.; Trivedi, R.; Lizakowski, M.; Rakoczy, S.; Brown-Borg, H. Multifactorial Attenuation of the Murine Heat Shock Response With Age. J. Gerontol. A. Biol. Sci. Med. Sci. 2020, 75, 1846-1852.

36. Nithianantharajah, J.; Barkus, C.; Murphy, M.; Hannan, A.J. Gene-environment interactions modulating cognitive function and molecular correlates of synaptic plasticity in Huntington's disease transgenic mice. Neurobiol. Dis. 2008, 29, 490-504.

37. Smith, G.A.; Rocha, E.M.; McLean, J.R.; Hayes, M.A.; Izen, S.C.; Isacson, O.; Hallett, P.J. Progressive axonal transport and synaptic protein changes correlate with behavioral and neuropathological abnormalities in the heterozygous Q175 KI mouse model of Huntington's disease. Hum. Mol. Genet. 2014, 23, 4510-4527.

38. Fourie, C.; Kim, E.; Waldvogel, H.; Wong, J.M.; McGregor, A.; Faull, R.L.M.; Montgomery, J.M. Differential Changes in Postsynaptic Density Proteins in Postmortem Huntington's Disease and Parkinson's Disease Human Brains. J. Neurodegener. Dis. 2014, 2014, 938530.

39. Carty, N.; Berson, N.; Tillack, K.; Thiede, C.; Scholz, D.; Kottig, K.; Sedaghat, Y.; Gabrysiak, C.; Yohrling, G.; Kammer, H. von der; et al. Characterization of HTT Inclusion Size, Location, and Timing in the zQ175 Mouse Model of Huntington's Disease: An In Vivo High-Content Imaging Study. PLoS One 2015, 10, e0123527.

40. Charych, E.I.; Akum, B.F.; Goldberg, J.S.; Jörnsten, R.J.; Rongo, C.; Zheng, J.Q.; Firestein, B.L. Activity-Independent Regulation of Dendrite Patterning by Postsynaptic Density Protein PSD-95. J. Neurosci. 2006, 26, 10164-10176.

41. Okabe, S.; Miwa, A.; Okado, H. Spine Formation and Correlated Assembly of Presynaptic and Postsynaptic Molecules. J. Neurosci. 2001, 21, 6105-6114.

42. Prange, O.; Murphy, T.H. Modular Transport of Postsynaptic Density-95 Clusters and Association with Stable Spine Precursors during Early Development of Cortical Neurons. J. Neurosci. 2001, 21, 9325-9333.

43. Smith, Y.; Raju, D.; Nanda, B.; Pare, J.-F.; Galvan, A.; Wichmann, T. The Thalamostriatal Systems: Anatomical and Functional Organization in Normal and Parkinsonian States. Brain Res Bull 2009, 78, 60-68.

44. Huerta-Ocampo, I.; Mena-Segovia, J.; Bolam, J.P. Convergence of cortical and thalamic input to direct and indirect pathway medium spiny neurons in the striatum. Brain Struct. Funct. 2014, 219, 1787-1800.

45. Jaeger, A.; Makley, L.; Gestwicki, J.; Thiele, D. Genomic heat shock element sequences drive cooperative human heat shock factor 1 DNA binding and selectivity. J. Biol. Chem. 2014, 289, 30459-30469.

46. Gylys, K.; Fein, J.; Yang, F.; Wiley, D.; Miller, C.; Cole, G. Synaptic changes in Alzheimer's disease: increased amyloid-beta and gliosis in surviving terminals is accompanied by decreased PSD-95 fluorescence. Am. J. Pathol. 2004, 165, 1809-1817.

47. Morimoto, R. Proteotoxic stress and inducible chaperone networks in neurodegenerative disease and aging. Genes Dev. 2008, 22, 1427-1438.

48. Ting, Y.K.; Morikawa, K.; Kurata, Y.; Li, P.; Bahrudin, U.; Mizuta, E.; Kato, M.; Miake, J.; Yamamoto, Y.; Yoshida, A.; et al. 
Transcriptional activation of the anchoring protein SAP97 by heat shock factor (HSF)- 1 stabilizes K v 1.5 channels in HL-1 cells. Br. J. Pharmacol. 2011, 162, 1832-1842.

49. Vallejo, D.; Codocedo, J.; Inestrosa, N. Posttranslational Modifications Regulate the Postsynaptic Localization of PSD-95. Mol. Neurobiol. 2017, 54, 1759-1776.

50. Riva, L.; Koeva, M.; Yildirim, F.; Pirhaji, L.; Dinesh, D.; Mazor, T.; Duennwald, M.L.; Fraenkel, E. Poly-glutamine expanded huntingtin dramatically alters the genome wide binding of HSF1. J. Huntingtons. Dis. 2012, 1, 33-45.

51. Yu, D.; Zarate, N.; Cuccu, F.; Yue, J.S.; Brown, T.G.; Tsai, A.; Mansky, R.; Jiang, K.; Kim, H.; Nanclares, C.; et al. Protein kinase CK2 alpha prime and alpha-synuclein constitute a key regulatory pathway in Huntington's disease. bioRxiv 2020, 2020.10.29.359380.

52. Kassubek, J.; Juengling, F.D.; Ecker, D.; Landwehrmeyer, G.B. Thalamic Atrophy in Huntington's Disease Co-varies with Cognitive Performance: A Morphometric MRI Analysis. Cereb. Cortex 2005, 15, 846-853.

53. Oakeshott, S.; Farrar, A.; Port, R.; Cummins-Sutphen, J.; Berger, J.; Watson-Johnson, J.; Ramboz, S.; Howland, D.; Brunner, D. Deficits in a Simple Visual Go/No-go Discrimination Task in Two Mouse Models of Huntington's Disease. PLoS Curr. 2013, 5.

54. Aron, A.; Watkins, L.; Sahakian, B.; Monsell, S.; Barker, R.; Robbins, T. Task-set switching deficits in early-stage Huntington's disease: implications for basal ganglia function. J. Cogn. Neurosci. 2003, 15, 629-642.

55. Nithianantharajah, J.; Hannan, A. Dysregulation of synaptic proteins, dendritic spine abnormalities and pathological plasticity of synapses as experience-dependent mediators of cognitive and psychiatric symptoms in Huntington's disease. Neuroscience 2013, 251, 66-74.

56. Milnerwood, A.J.; Raymond, L.A. Early synaptic pathophysiology in neurodegeneration: insights from Huntington's disease. Trends Neurosci. 2010, 33, 513-523.

57. Ran, F.A.; Hsu, P.D.; Wright, J.; Agarwala, V.; Scott, D.A.; Zhang, F. Genome engineering using the CRISPR-Cas9 system. Nat. Protoc. 2013811 2013, 8, 2281-2308. 